\title{
Linx
}

Revue des linguistes de l'université Paris X Nanterre

64-65 | 2011

Les genres de discours vus par la grammaire

\section{Un genre peut en cacher un autre : une histoire de détournement}

Sylvie Freyermuth

\section{(2) OpenEdition}

Journals

Édition électronique

URL : http://journals.openedition.org/linx/1411

DOI : 10.4000/linx.1411

ISSN : 2118-9692

Éditeur

Presses universitaires de Paris Nanterre

\section{Édition imprimée}

Date de publication : 1 juillet 2011

Pagination : 173-187

ISSN : 0246-8743

\section{Référence électronique}

Sylvie Freyermuth, «Un genre peut en cacher un autre : une histoire de détournement », Linx [En ligne], 64-65 | 2011, mis en ligne le 01 juillet 2014, consulté le 19 avril 2019. URL : http:// journals.openedition.org/linx/1411; DOI : 10.4000/linx.1411 


\title{
Un genre peut en cacher un autre : une histoire de détournement
}

\author{
Sylvie Freyermuth \\ Université du Luxembourg - FLSHASE, UR LCMI \\ Professeur associé au Centre Écritures, Université de Lorraine, E.A. 3943 \\ Correspondant étranger du Centre de Recherche sur l'Imaginaire, Université \\ Grenoble 3, E.A. 610 \\ Sylvie.Freyermuth@uni.1u-sylviefreyermuth@orange.fr
}

\section{Introduction}

Le reproche que l'on peut adresser aux divers champs qui appréhendent les genres de discours, qu'il s'agisse de l'analyse textuelle, de l'analyse du/des discours ou encore des travaux sur corpus, concerne, mis à part quelques travaux ponctuels, le choix de privilégier la catégorie du genre par rapport à l'analyse des faits de langue, même si ceux-ci font l'objet d'une étude approfondie. À l'inverse, les théories syntaxiques témoignent aux faits de langue un intérêt premier, mais «oublient» la plupart du temps l'influence des genres dans lesquels ils s'inscrivent. En d'autres termes, les uns et les autres, en séparant ces deux pôles, négligent de s’interroger sur la relation d'influence dynamique susceptible de les unir.

\section{Faits de langue, genres et interrelations}

Je me propose d'envisager cette question en posant l'analyse des faits de langue comme préalable à l'étude d'un texte littéraire extrait d'un roman contemporain, me 
conformant ainsi à la division qu'opère Bakhtine entre «genres simples » et «genres complexes », les second intégrant les premiers ${ }^{1}$. Si l'on se réfère à ces deux types de genres du discours ainsi définis, et surtout au phénomène d'absorption de l'un par l'autre, il me semble pertinent d'évoquer le principe du continum développé par $\mathrm{Adam}^{2}$ à partir de la théorie de Bally de laquelle le linguiste dégage cinq principes ; ceux-ci défendent l'idée de l'existence d'une communauté linguistique matricielle (la langue maternelle comme trésor commun à tous), qui se différencie selon que le locuteur vise une communication utilitaire ou, au contraire, la création d'une œuvre esthétique ${ }^{3}$. Les genres du discours premiers (simples), «qui se sont constitués dans les circonstances d'un échange verbal spontané» (Bakhtine, 1984 : 267) pourraient s'apparenter à la langue ordinaire dont parle Bally, non exempte de créativité mais tournée essentiellement vers l'usage pratique. Quant aux genres seconds (complexes), qui «apparaissent dans les circonstances d'un échange culturel $[\ldots]$ plus complexe et relativement plus évolué » (Bakhtine, 1984: 267), ils naissent d'une intention esthétique et, comme l'affirme Adam (1997: 55), «l'art littéraire se caractérise par une transformation des intentions pratiques en intentions esthétiques, le moyen se transforme en but». Cinq ans après Le style dans la langue, Adam $(2002: 78)^{4}$ ne varie pas, puisque il cite Bally en ces termes :

Il est temps de ne plus considérer la langue littéraire comme une chose à part, une sorte de création ex nibilo; elle est avant tout une transposition spéciale de la langue de tous; seulement les motifs biologiques et sociaux de cette langue deviennent motifs esthétiques. (1965a: 62)

Je discerne un lien de familiarité entre la théorie de Bally et celle de Bakhtine et associe l'usage commun de la langue défini par le premier linguiste au genre du discours simple caractérisé par le second; de même, le discours orienté vers un but esthétique (Bally) s'apparente au genre du discours second de Bakhtine, en ce qu'il intègre des structures propres au genre du discours premier à un échange mené dans « les circonstances d'un échange culturel ». Cette absorption du discours premier par le discours second se fait dans une langue qui évolue sur la trajectoire d'un continumm d'un pôle à l'autre (phénomène de transmutaion chez Bakhtine) ; autrement dit, pour reprendre l'analyse d'Adam (1997 et 2002), la langue littéraire est à la fois familière et étrangère à la langue commune, puisqu'elle s'en distingue par son intentionnalité et

\footnotetext{
1 « Il importe à ce point de prendre en considération la différence essentielle qui existe entre le genre du discours premier (simple) et le genre du discours second (complexe). Les genres seconds du discours - le roman, le théâtre, le discours scientifique, le discours idéologique, etc. - apparaissent dans les circonstances d'un échange culturel (principalement écrit) - artistique, scientifique, socio-politique plus complexe et relativement plus évolué. Au cours du processus de leur formation, ces genres seconds absorbent et transmutent les genres premiers (simples) de toutes sortes, qui se sont constitués dans les circonstances d'un échange verbal spontané. » (Bakhtine, M., 1984 : 267).

2 Adam, J.-M., 1997 : 55 et sq.

3 J.-M. Adam (2002 : 77) déclare : « Pour compléter cette conception d'un continu, j’ai proposé, dans Le style dans la langue (1997, p.46-84), de distinguer, chez Bally, trois ordres de manifestation de la totalité de la langue : la langue naturelle, la langue littéraire et le style ».

${ }^{4}$ Voir note précédente.
} 
s'en rapproche par le fonds qu'elle partage avec elle. C'est pourquoi il me semble recevable, au premier abord, d'entrer dans l'analyse des genres du discours à travers l'observation des faits de langue, qui ne sont perceptibles qu'actualisés dans un énoncé singulier. Cette idée d'ambivalence est proche de celle qui sous-tend la pensée de Bakhtine, à savoir que tout énoncé est coulé dans le moule du genre du discours dans lequel il s'inscrit, et qu'en même temps, la nature du genre du discours est génératrice d'un certain type d'énoncés. Bakhtine (1984: 268) affirme en effet: "La langue pénètre dans la vie à travers des énoncés concrets (qui la réalisent), et c'est encore à travers des énoncés concrets que la vie pénètre dans la langue ».

Dès lors - et la relecture du chapitre «Les genres du discours » in Esthétique de la création verbale ne m'a pas contredite - le lien entre énoncé, genre du discours et style m'est apparu d'une grande conséquence. Tout comme Bally, Bakhtine associe le style au caractère individuel de l'énoncé et admet qu'il existe des degrés dans l'empreinte stylistique d'un énonciateur :

Le style est indissolublement lié à l'énoncé et à des formes typiques d'énoncés, c'està-dire à des genres du discours. L'énoncé (oral et écrit) - premier et second [...] - est individuel, en vertu de quoi il peut refléter l'individualité de celui qui parle (ou écrit). [...] Mais tous les genres ne sont pas également aptes à refléter une individualité dans la langue de l'énoncé, autrement dit, propices au style individuel. (Bakhtine, 1984 : 268)

De fait, la littérature apparaît comme le lieu le plus propice à l'expression d'un style individuel. Si bien qu'une approche stylistique des genres du discours à travers l'énoncé, dont Bakhtine précise bien qu'il doit être saisi dans un tout, me parait pertinente. Bakhtine estime en effet que « la grammaire et la stylistique se rejoignent et se séparent dans tout fait concret qui, envisagé du point de vue de la langue, est un fait de grammaire, envisagé du point de vue de l'énoncé individuel, est un fait stylistique. $[\ldots]$ Ces deux points de vue [...] doivent se combiner organiquement (avec le maintien méthodologique de leur différence) sur la base de l'unité réelle que représente le fait de langue. » (Bakhtine, 1984 : 272) Dans son article de 2002, Adam, en s'appuyant sur les théories du "Cercle de Bakhtine », défend l'idée d'un dépassement des frontières qui séparent la grammaire de la stylistique et qui se réaliserait dans une «translinguistique » :

Situant l'acte stylistique' dans l'énonciation même, ils proposent une 'synthèse dialectique' des points de vue de l'objectivisme abstrait' grammairien et du 'subjectivisme individualiste' stylistique (Bakhtine-Volochinov 1977 : 118). (Adam, $2002: 82)$

Et plus loin :

De notre point de vue, il est impossible et méthodologiquement irrationnel d'établir une frontière stricte entre la grammaire et la stylistique, entre le schéma grammatical et sa variante stylistique. Cette frontière est instable dans la vie même de la langue [...]. (Bakhtine-Volochinov 1977 : 174) (Adam, $2002: 82$ )

Il m'apparaît alors indispensable d'entrer dans une analyse stylistique à travers l'étude des faits de langue saillants. 
Sylvie Freyermuth

Par sa nature, l'exemple littéraire que j'ai choisi ${ }^{5}$ pour objet d'étude relève, selon Bakhtine, d'un genre de discours second ; mais il comporte manifestement des traits propres à un genre de discours premier, par lequel je demande à présent au lecteur de bien vouloir consentir un détour, car les nécessités de la démonstration me commandent d'entamer mon propos par lui.

\section{Du genre premier au genre second}

Je procèderai en optant délibérément pour une stylistique compositionnelle, c'est-à-dire une méthode qui emprunte aux domaines de la linguistique (syntaxe et sémantique), de la pragmatique, de la rhétorique et de l'analyse textuelle ${ }^{6}$. La combinaison de ces différents niveaux doit mettre en évidence un faisceau de faits de langue directement impliqués dans l'effet produit sur le lecteur.

\subsection{Aux fourneaux}

Soit le texte suivant :

(1) Simplicité marine

Choisissez une douzaine de belles coquilles Saint-Jacques déjà décortiquées. Épongezles dans un papier absorbant et réservez-les. Ciselez de la ciboulette et du basilic frais et réservez, lavez quelques feuilles de menthe que vous garderez entières, taillez un citron en très fines rondelles que vous couperez en 4. Préparez la décoration de votre plat de présentation en y plaçant de la mâche assaisonnée de vinaigre balsamique et des tomates-cerises confites.

Enduisez d'un peu d'huile d'olive une poêle et faites-la chauffer à feu vif. Lorsque la poêle est bien chaude, saisissez les coquilles Saint-Jacques des deux côtés. Elles doivent être dorées. Saupoudrez-les de sucre afin de les caraméliser, salez et poivrez, ajoutez un soupçon de gingembre moulu. Disposez les coquilles sur votre plat, arrosez-les d'un trait d'huile d'olive et de jus de citron, répartissez les fines herbes ciselées que vous avez réservées et terminez la décoration avec les feuilles de menthe et les quarts de rondelles de citron. Servez aussitôt.

Ce texte d'une douzaine de lignes est dominé par l'absence de la première personne explicite et la présence massive de l'acte injonctif : 20 verbes sont à l'impératif à valeur d'inaccompli. En outre, l'emploi du futur se charge également d'une valeur injonctive :

(1bis) : lavez quelques feuilles de menthe que vous garderę, entières.

est l'équivalent de

\footnotetext{
${ }^{5}$ Rouaud, J., Des hommes illustres, Paris, Les Éditions de Minuit, 1993, p. 37-39.

${ }^{6}$ Je n'entrerai pas ici dans les débats actuels sur le bien-fondé ou non de l'interdisciplinarité et moins encore dans les distinctions, parfois délicates, opérées entre trans-/inter-/multidisciplinarité. Une littérature abondante existe à ce sujet, par exemple : Després, C., Lawrence, R. J., 2004 : 397-405 ; Beetham, G., Fernández, M., 2010 : 7-13 ; Huutoniemi, K., Thompson-Klein, J., Bruun, H., Hukkinen, J., $2010: 79-88$.
} 
(1ter) : lavez quelques feuilles de menthe et gardez-les entières.

Il en va de même pour «taillez un citron en très fines rondelles que vous couperez en 4. » Le gérondif « en y plaçant » établit un lien corrélatif entre l'injonction «Préparez la décoration de votre plat de présentation » et la manière de procéder décrite (ajout de mâche et tomates-cerises confites). L'analyse est identique pour l'infinitif dans la proposition «afin de les caraméliser». Enfin, le sens du verbe devoir dans l'expression "elles doivent être dorées » indique qu'ordre est donné de saisir les coquilles Saint-Jacques jusqu'à ce qu'elles aient atteint ce degré de cuisson. Au total, les rares verbes qui échappent à la modalité injonctive sont sémantiquement des résultats de l'exécution de l'ordre donné : «Lorsque la poêle est bien chaude » qui découle de «Enduisez d'un peu d'huile d'olive une poêle et faites-la chauffer à feu vif», et la relative «[les fines herbes ciselées] que vous avez réservées» est anaphoriquement liée à «Ciselez de la ciboulette et du basilic frais et réservez ».

Deuxième point, à présent, lié aux forces illocutoire et perlocutoire de l'impératif: la première personne n'apparaît pas explicitement, mais indirectement comme source des consignes données; ce qui provoque une forte implication de l'allocutaire, sommé de s'exécuter: les occurrences de la deuxième personne correspondent évidemment à la majorité des formes verbales employées. Enfin, les pronoms représentants qui assurent le chaînage anaphorique renvoient le plus souvent à des entités qui occupent une position de complément. Par exemple : "Ciselez de la ciboulette et du basilic frais [...]. [T] aillez un citron en très fines rondelles que vous couperez en 4 ».

Troisièmement, on notera que les informations données se limitent à la stricte nécessité de l'exécution : ingrédients, mode de traitement, présentation. Elles répondent en cela aux maximes conversationnelles élaborées par Grice ${ }^{7}$ (quantité, qualité, relation et modalité), mais tout particulièrement à la première d'entre elles :

La catégorie de QUANTITÉ concerne la quantité d'information qui doit être fournie, et on peut y rattacher les règles suivantes :

1. Que votre contribution contienne autant d'information qu'il est requis (pour les visées conjoncturelles de l'échange). [...]

2. Que votre contribution ne contienne pas plus d'information qu'il n'est requis. (Grice, 1979, p. 61)

Pour terminer, les objets mis en scène dans le genre discursif que je viens de décrire confirment que nous avons affaire à une utilisation de la langue qui relève de la sphère du quotidien : la recette de cuisine. Dans cette étude comparée, il s'agit d'un choix personnel; il aurait été possible, en effet, de recourir à un autre texte de type procédural $^{8}$ tel qu'une notice de montage, une consigne de sécurité etc. ${ }^{9}$ Quoi qu'il en soit, selon Jacques et Poibeau, les recettes de cusine «font typiquement partie de ce que la littérature linguistique et psycholinguistique analyse généralement comme textes

${ }^{7}$ Grice, H. P., 1975 : $41-58$; traduction, 1979: 57-72.

8 Pour cette question, voir par exemple Adam, J.-M., [1992] - 2001.

9 Jacques, M.-P., Poibeau, Th., 2010, pn.p. 
procéduraux. Ce sont les parangons de la catégorie, ceux qui en forment le cœur.» (2010, n.p.) La seule réserve que l'on pourrait émettre quant à l'exemple 1 vise le titre, dont la coquetterie n'est pas un paramètre caractéristique du genre procédural ${ }^{10}$.

\subsection{Les recettes du métier}

Envisageons à présent l'extrait du roman de Jean Rouaud (cité supra) dont la nature même le classe dans la catégorie bakhtinienne du genre de discours second, ici littéraire. Nous y reconnaitrons les traits caractérisant le genre procédural ${ }^{11}$, qui n'est pourtant, en l'occurrence, ni une recette de cuisine ni un ensemble de consignes donnés par un personnage de la fiction romanesque ; en revanche, les propriétés du texte procédural présentes dans la structure même de l'énoncé de l'auteur-narrateur intriguent le lecteur parce que celui-ci perçoit, à travers elles, la nécessité de s'interroger sur le dessein même de l'énonciateur. On sera alors amené à questionner l'efficacité d'une typologie des faits de langue dans leur fonction de prédictibilité des genres du discours.

(2) ${ }^{12}$ Maintenant, empilez dans une valise cubique adaptée une cinquantaine d'assiettes aux motifs différents, soulevez, traversez la rue, poussez la porte du magasin, déposez, dénouez la sangle de cuir qui ceinture l'ensemble et prévient tout risque d'effondrement, déballez, exposez, faites l'article, subissez les mimiques du marchand qui de très loin prépare ainsi son refus et ne tient pas à ce qu'un émerveillement de sa part amène un malentendu. Remballez sans soupirer. Resoulevez, retournez à la case départ. Renouvelez l'opération. Sortez cette valise pleine de verrerie avec ses cases de velours rouge, cette autre de bibelots sommairement enveloppés dans des carrés de tissu et cette autre encore, et celle-là que vous avez oubliée dont vous assurez, connaissant son goût, qu'elle va intéresser le client. Dites : je reviens. Revenez vite, le bras à demi arraché par la presque-malle que vous avez bien du mal à ne pas traîner. Ne montrez rien de votre lassitude. Expliquez : premier choix, second choix, promotion, prix de lancement, prix imposé, limite des stocks. Faites sonner, d'une pichenette de l'ongle sur son rebord, la splendeur en cristal taillé qui devra recevoir les fruits ou ce qu'on voudra. Demandez des nouvelles des uns et des autres. Compatissez. Ne vous appesantissez pas. Déviez. Prenez position sur le temps, dites : ça va s'arranger. Quand votre interlocuteur avance que la vie est dure en ce moment pour la profession, contrez: c'est général. Le cahier de

\footnotetext{
${ }^{10}$ Il s'agit d'une recette que j’ai élaborée et qui se contente de décrire le protocole de confection du plat.

11 À partir d'annotations de lecteurs sur un corpus, Jacques et Poibeau (2010, n.p.) ont défini des propriétés prototypiques de ce genre de textes parmi lesquelles on notera les «marques d'injonction», les «marques de condition» et «la phraséologie propre au domaine». Il existe cependant des segments « à la marge », c'est-à-dire qu'ils prêtent à discussion. Selon ces linguistes, les recettes sont un type de texte prototypique du genre procédural: "Cette catégorie regroupe, notamment, les modes d'emploi, les notices explicatives, les manuels et les guides d'utilisation, les consignes de sécurité, les recettes de cuisine, les do-lists utilisés en aéronautique, etc. [...]. »

12 Le lecteur voudra bien me pardonner la longueur, nécessaire, de l'extrait cité.
} 
commandes est prêt posé en équilibre sur un amoncellement d'échantillons, la couverture et les pages déjà remplies retournées sur l'envers. Glissez deux feuilles de papier carbone sous l'original de façon à le reproduire en deux autres exemplaires : un pour le grossiste de Quimper, l'autre pour le détaillant, le troisième pour vous. Sortez le stylo à bille dont vous appréciez qu'il ne tache pas vos poches comme le stylo à encre, notez en respectant bien les colonnes : numéro de référence de l'article, intitulé, prix unitaire, quantité (vous ferez le calcul, ce soir, à l'hôtel). Dites : je vous écoute. Deux modèles comme ceci, un autre comme celui-là, trois de cette sorte. C'est tout? C'est tout pour aujourd'hui. Dissimulez votre amertume que pour si peu la matinée ait été perdue. Remerciez, remballez, saluez. Au prochain passage. (Rouaud, J., Des bommes illustres, 1993, p. 37-39)

La lecture de cet extrait permet de relever des faits de langue de même nature que ceux présents dans les textes procéduraux, notamment la recette commentée supra: une présence massive des formes à l'impératif d'aspect inaccompli (on dénombre 36 verbes à ce mode), la présence du futur catégorique ("vous ferez le calcul, ce soir, à l'hôtel»), d'autres formes verbales dont la valeur injonctive est le résultat de celle des impératifs dont elles dépendent («Dites : je vous écoute »), autant de formes dont l'objectif est de faire faire ou d'interdire de faire ${ }^{13}$. Ce morceau de bravoure, tel qu'on en rencontre dans le roman roualdien au détour d'une page, ressemble fort à un vademecum du parfait représentant de commerce contenant les « recettes du métier». Il est ainsi remarquable que la série de consignes se fractionne en microséquences délimitées par la ponctuation. La première phrase, qui compte en près de 5 lignes 10 verbes à l'impératif, condense les gestes de toute la phase de la représentation des produits qui doit conduire à la commande du commerçant. Cet effort entièrement tendu vers l'espoir de réaliser un bon chiffre d'affaires se lit dans la longueur de la phrase qui progresse grâce à la juxtaposition. Par contraste, les 3 phrases suivantes (Remballez, sans soupirer. Resoulevez, retournez à la case départ. Renouvelez. l'opération.) indiquent autant de micro-actes : le remballage et le masquage de la déception, puis le retour vers la voiture (dont les deux verbes, par leur sens réitératif re-soulever, retourner - font pendant aux deux premières lignes du passage), et enfin, la proposition minimale qui indique elle aussi une répétition ad libitum, mais dont la fonction résomptive anaphorise en un macroacte l'ensemble des micro-actes décrits. Conformément au premier texte, les maximes conversationnelles ne sont pas violées, puisque les gestes à accomplir pour mériter le label de bon représentant en porcelaine et cristaux sont, avec rationalité, clairement et scupuleusement énoncés. S'agit-il pour autant d'une recette?

13 On mentionnera la citation de Heurley que font Jacques et Poibeau (2010, n.p.) : «La première caractéristique des textes procéduraux est qu'ils communiquent à la fois des informations déclaratives et procédurales [...]. Les informations déclaratives correspondent à toutes les informations qui ne sont pas directement orientées vers l'action. Ces informations sont présentes dans les textes procéduraux comme dans toutes les autres catégories textuelles. [...] Les informations procédurales spécifient, quant à elles, des opérations ou des actions que l'utilisateur doit exécuter (instructions positives), ou au contraire, s'abstenir d'exécuter (instructions négatives). (Heurley, 2001 : 30-31)». 


\section{Genre détourné, effet masqué}

Les références de l'extrait étudié indiquent évidemment clairement qu'il s'agit d'un texte romanesque, qui relève donc d'un genre de discours second selon Bakhtine, à savoir le discours littéraire, celui-là même dont Bally ([1913], 1965 et [1909], 1951) estimait qu'il ne devait pas être un domaine d'investigation privilégié, dans la mesure où il est perçu comme le résultat d'une accumulation de tous les éléments de style hérités des générations successives, la cristallisation, en quelque sorte, de la norme haute d'une société à une époque donnée, discours littéraire dont Mainguenau (2003) affirmait encore qu'il était - à tort - sacralisé.

Bien qu'on ait relevé des similitiudes entre les textes des exemples 1 et 2, y compris dans la mise au premier plan, par l'usage du mode injonctif, de l'instance de l'allocutaire, certains indices invitent le lecteur à se diriger vers une autre interprétation que celle de la recette stricto sensu, et de ce fait à s'interroger sur l'effet produit par le recours de l'auteur à un tel procédé.

Tout d'abord, des segments textuels indiquent clairement l'existence d'un narrateur omniscient qui dispose d'informations, notamment psychologiques, liées au référent de la forme de l'allocutaire : «et celle-là que vous avez oubliée dont vous assurez, connaissant son goût, qu'elle va intéresser le client»; "Sortez le stylo à bille dont vous appréciez, qu'il ne tache pas vos poches comme le stylo à encre ». Le référent est bien ici le père, et non pas le lecteur : les formes concernées sont déclaratives et non injonctives.

Le passage suivant de discours représenté au style direct libre ${ }^{14}$, comme il en existe fréquemment dans la prose roualdienne ${ }^{15}$, est ambigu : «Dites : je vous écoute. Deux modèles comme ceci, un autre comme celui-là, trois de cette sorte. C'est tout ? C'est tout pour aujourd'hui. » Il peut s'agir d'une injonction, le narrateur donnant un conseil de protocole à observer lors d'une transaction commerciale. Mais les informations sur le nombre d'objets commandés montrent également qu'il s'agit de la description d'une situation actualisée, fût-ce au niveau diégétique. Il en va de même pour l'échange final qui marque la déception du représentant de commerce, et pour la proposition entre parenthèses «(vous ferez le calcul, ce soir, à l'hôtel)» qui peut signifier deux choses : au plan du genre procédural, le locuteur conseille à l'allocutaire de ne pas perdre de temps à faire le calcul chez le commerçant; au plan fictionnel, le narrateur (omniscient) connait les habitudes de son personnage.

Cette oscillation entre deux possibilités interprétatives du texte - le genre procédural et la description - atypique - des conditions de travail harassantes du père de l'auteur-narrateur, Joseph Rouaud, voyageur de commerce - pousse le lecteur à s'interroger sur le choix esthétique de Jean Rouaud. Pourquoi préférer le genre du texte procédural (la recette) à celui de la narration incluant la troisième personne en lieu et place de la deuxième, comme en témoigne un extrait du passage du roman remanié par mes soins ?

\footnotetext{
14 Je nomme ainsi les formes de discours direct non signalé par des guillemets.

${ }^{15}$ Cf. Freyermuth, S., 2006, Jean Rouaud et le périple initiatique : une poétique de la fluidité, Paris, Budapest, Turin, L'Harmattan, Coll. « Critiques littéraires ».
} 
(2bis) [Maintenant, il empile dans une valise cubique adaptée une cinquantaine d'assiettes aux motifs différents, il soulève, traverse la rue, pousse la porte du magasin, dépose, dénoue la sangle de cuir qui ceinture l'ensemble et prévient tout risque d'effondrement, il déballe, expose, fait l'article, il subit les mimiques du marchand qui de très loin prépare ainsi son refus et ne tient pas à ce qu'un émerveillement de sa part amène un malentendu. Il remballe sans soupirer. Il resoulève, retourne à la case départ. Il renouvelle l'opération. Il sort cette valise pleine de verrerie [...], et celle-là qu'il a oubliée dont il assure, connaissant son goût, qu'elle va intéresser le client. Il dit: je reviens. Il revient vite, le bras à demi arraché par la presque-malle qu'il a bien du mal à ne pas traîner. Il ne montre rien de sa lassitude. [...] Il glisse deux feuilles de papier carbone sous l'original de façon à le reproduire en deux autres exemplaires: un pour le grossiste de Quimper, l'autre pour le détaillant, le troisième pour lui. Il sort le stylo à bille dont il apprécie qu'il ne tache pas ses poches comme le stylo à encre, il note en respectant bien les colonnes : numéro de référence de l'article, intitulé, prix unitaire, quantité (il fera le calcul, ce soir, à l'hôtel). Il dit : je vous écoute. Deux modèles comme ceci, un autre comme celui-là, trois de cette sorte. C'est tout? C'est tout pour aujourd'hui. Il dissimule son amertume que pour si peu la matinée ait été perdue. Il remercie, remballe, salue. Au prochain passage.] (d'après Jean Rouaud, Des hommes illustres, p. 37-39)

On note tout d'abord, dans cette transposition, l'emploi du présent de l'indicatif, rendu obligatoire par la présence de l'adverbe embrayeur temporel maintenant ${ }^{16}$ et de l'expression ce soir. De plus, le chaînage anaphorique assumé par le clitique de $3^{e}$ personne $i l$ et ses corrélats (déterminants possessifs), employés exclusivement pour dénoter le père, produit un effet fortement cohésif ${ }^{17}$. Combiné au mode assertif, ce procédé confère au texte une densité qui permet de représenter le personnage principal du passage dans la constance de ses actions, dans leur déroulement inexorable donné à voir au lecteur. Mais en l'occurrence, cette distanciation créée par la perception d'une troisième personne bien distincte de l'instance de l'allocutaire transforme ce dernier en une sorte de voyeur, dont l'impuissance risque de le rendre distant par rapport au personnage central.

\subsection{Divers modes de sollicitation du pathos}

Revenons à présent à l'option de l'auteur-narrateur Jean Rouaud, soit le texte de l'exemple 2. Bien que le lecteur repère très aisément tous les faits de langue caractéristiques du genre procédural, il n'empêche qu'il se trouve devant le cas décrit par Bakhtine d'un genre de discours simple (la recette) transposé dans un genre de discours second (le roman). Prend-il alors cet extrait comme tel, se contentant d'y reconnaitre le respect des maximes de quantité et de qualité l'incitant à exécuter une action? J'en doute, car la succession des consignes à l'impératif n'atteint pas un

\footnotetext{
${ }^{16}$ L'auteur est coutumier de ces énallages temporels qui ont également une fonction à remplir, comparable à celle de la présence de l'instance de la deuxième personne. Cf. Freyermuth, S., 2006.

${ }^{17}$ Pour cette question, voir Freyermuth, S., 2007 ; Kleiber, G., 1994 ; Kleiber, G., 1997 ; Kleiber, G., 2002.
} 


\section{Sylvie Freyermuth}

allocutaire intégré au récit, mais place le destinataire à la frontière de la fiction à laquelle appartient l'histoire, et du réel dans lequel s'ancre le lecteur. Ainsi, l'auteurnarrateur agit sur ce dernier dans une intention pragmatique, le convoque dans sa narration comme instance agissante, provoque son identification à Joseph Rouaud et orchestre son apprentissage du rude métier de représentant de commerce. D’ailleurs, là où le chaînage anaphorique de ma version remaniée (exemple 2bis) ajustait l'attention du lecteur sur le personnage de Joseph en plein exercice de son art, ce sont les actions elles-mêmes que l'impératif met dans le focus au sein du texte originel. Le rythme de ce passage est à dessein particulièrement soutenu, réussissant à donner le vertige : l'éreintante manutention est mise en évidence par une fréquente réduction de la phrase complexe à des propositions juxtaposées ; par exemple :

Maintenant, empilez dans une valise cubique adaptée une cinquantaine d'assiettes aux motifs différents, soulevez, traversez la rue, poussez la porte du magasin, déposez, dénouez la sangle de cuir qui ceinture l'ensemble et prévient tout risque d'effondrement, [...]. (Rouaud, J., 1993, p. 37)

Les propositions peuvent être ramenées elles-mêmes à leur plus simple expression par la seule mention du procès, qui constitue alors une phrase: «Compatissez. Ne vous appesantissez pas. Déviez.» Ou encore, cette phrase au rythme ternaire «Remerciez, saluez, remballez. », portée par la juxtaposition de 3 impératifs de 3 syllabes chacun, et rimant tous en $[\mathrm{e}]^{18}$, donne une impression de ballet servile. L'allitération produit également un rythme et imprime au passage un air de litanie désespérante, comme c'est le cas dans l'exemple suivant, scandé par le son [R] : «Remballez sans soupirer. Resoulevez, retournez à la case départ. Renouvelez l'opération. ${ }^{19}$ " L'assonance n'est pas en reste non plus dans les impératifs de cet exemple : [ã $-\mathrm{a}-\mathrm{e}$ (Remballez) ; [ə - u - (ə) - e] (Resoulevez) ; [ə - u - e] (retournez); [ə-u - (ə) - e] (Renouvelez). Ces procédés phoniques contribuent à souligner des microséquences qui en disent long sur la déception de Joseph Rouaud. Par exemple, le contraste de la phrase minimale « Remballez sans soupirer. » (impératif et infinitif négatif dans un rôle adverbial de modifieur) avec la phrase de près de 5 lignes qui précède ${ }^{20}$, montre à quel point le personnage doit ravaler sa lassitude face à cette disproportion entre l'effort fourni et le maigre résultat atteint. La convergence de tous ces effets ${ }^{21}$ n'est pas anodine car elle éveille l'attention du lecteur quant à la possible intention ${ }^{22}$ de l'auteur-narrateur, à savoir la mise en œuvre d'une stratégie. En

\footnotetext{
18 On notera que les verbes ayant la finale en [e] sont au nombre de 32 sur 36, ce qui confère au texte un tempo indéniable.

${ }^{19}$ Je souligne par l'italique.

20 C'est ici une caractéristique de l'écriture roualdienne, dont l'oscillation entre les rythmes amples (expansion) et brefs (contraction) miment une pulsation. A ce sujet, voir Freyermuth, S., 2010 et Freyermuth, S., 2011.

21 Je rappelle la nécessité de recourir à différents champs d'analyse en fonction de la variété des procédés employés : pragmatique, rhétorique, poétique, syntaxe, sémantique, narratologie.

22 Il est délicat de se préoccuper de l'intention de l'auteur, dont on ne peut avoir d'assurance qu'à travers ses commentaires sur sa propre œuvre ou en estimant, comme Riffaterre (1959), qu'une convergence de faits de langue en un faisceau pertinent sont les signes d'une intention.
} 
effet, sans laisser à son lecteur le temps de souffler et en s'adressant à lui sur le mode impératif, Jean Rouaud fait agir une force illocutoire et perlocutoire qui place l'allocutaire devant une alternative : s'exécuter ou refuser de le faire, ce qui concède à ce dernier une liberté de choix dont il doit seul évaluer les conséquences. De fait, lorsque dans la première partie de cet extrait, l'auteur-narrateur a donné au lecteur en neuf lignes l'ordre d'exécuter treize gestes pénibles, dont dix rien que dans la première phrase, parvenu à «Renouvelez l'opération », celui-ci songe à se révolter et à refuser d'obtempérer, tant il est déjà éprouvé et las de répéter quasi mécaniquement ces gestes identiques sans la moindre garantie de résultat. Pragmatiquement, le pathos du lecteur a été sollicité de manière efficace.

\subsection{Le lecteur : un acteur sensible}

Non content de transformer son lecteur en son propre interlocuteur, voire en son exécutant, l'auteur-narrateur l'attire dans la diégèse, dans une espèce de théâtralisation de la situation, puisque à travers ce procédé, il le fait converser avec les commerçants démarchés, grâce à une maîtrise virtuose de la polyphonie énonciative ${ }^{23}$, dont Fløttum ${ }^{24}$ estime qu'elle pourrait ouvrir des horizons prometteurs au domaine énonciatif des typologies existantes, comme celle élaborée par Adam. Par exemple :

Quand votre interlocuteur avance que la vie est dure en ce moment pour la profession, contrez : c'est général.

L'auteur-narrateur est le locuteur qui fait entendre deux énonciateurs ${ }^{25}$ par le truchement des discours représentés : le commerçant à la source de l'idée «la vie est dure en ce moment pour la profession», et l'auteur-narrateur qui indique au lecteurreprésentant de commerce la réponse «c'est général ». Mais en même temps, un autre énonciateur est indissolublement lié à cette voix, la personne de Joseph Rouaud dont le quotidien est décrit par le passage.

L'exemple suivant est plus complexe :

Dites : je vous écoute. Deux modèles comme ceci, un autre comme celui-là, trois de cette sorte. C'est tout? C'est tout pour aujourd'hui.

Dans cet extrait, les configurations sont multiples : l'auteur-narrateur peut prendre en charge la totalité de l'énoncé, en mettant en scène les différents rôles: le commerçant démarché, le père, le lecteur/représentant occasionnel. Mais il est possible que Joseph soit l'énonciateur de la totalité de l'énoncé, hormis «dites», et dans ce cas, il répète les propos du commerçant ; dernière solution : on peut y voir la reproduction d'un dialogue commerçant / Joseph :

\footnotetext{
23 On peut indiquer l'intéressant ouvrage, Perrin, L. (éd.), 2006.

${ }^{24}$ Pour cette question, voir Fløttum, K., 1999 ; Fløttum, K., 2002-a ; Fløttum, K., 2002 ; Fløttum, K., 2002-b.

${ }^{25}$ Il s'agit ici de la distinction que Ducrot opère entre le locuteur, à savoir celui qui prend en charge l'acte locutoire, et l'énonciateur qui consiste en la source d'un énoncé. Voir Ducrot, O., 1984.
} 
Sylvie Freyermuth

Auteur-narrateur : \{ Dites [auteur-narrateur] : je vous écoute [Joseph]. Deux modèles comme ceci, un autre comme celui-là, trois de cette sorte [commerçant]. C'est tout ? [Joseph] C'est tout pour aujourd'hui. [commerçant] ou [Joseph] ou [auteur-narrateur] \}

Il ne faut pas perdre de vue non plus que tout énoncé pris en charge par Joseph peut l'être aussi par le lecteur, et ce justement grâce à la force illocutoire et perlocutoire de l'impératif. Quant au locuteur d'énoncé (en l'occurrence l'auteur-narrateur), il intervient aussi bien, selon Fløttum ${ }^{26}$, à l'aide de son propre point de vue que d'autres points de vue : il est «le metteur en scène du jeu polyphonique présenté ». Une complexité de cette nature n'est évidemment pas la caractéristique déterminante du genre de discours dans lequel entre la recette, mais pourrait au contraire constituer une qualité discriminante. En procédant de la sorte, en faisant endosser le costume de représentant de commerce de Joseph Rouaud à son lecteur, durant ne fût-ce que la moitié d'une de ses interminables journées, l'auteur-narrateur fait partager à celui-ci la vie, les espoirs et déceptions de son père, comme en témoigne la phrase «Dissimulez votre amertume que pour si peu la matinée ait été perdue.»D'autres faits qui dépassent les propriétés du genre de discours procédural auquel appartient la recette le confirment. Ainsi :

Dites : je reviens. Revenez vite, le bras à demi arraché par la presque-malle que vous avez, bien du mal à ne pas traîner. ${ }^{27}$

L'apposition «le bras à demi arraché » ne peut pas entrer dans le domaine de l'injonction - à moins qu'il s'agisse d'une feinte pour donner au commerçant l'image d'un travailleur s'épuisant à la tâche et ainsi exciter sa pitié, et dans ce cas, il faudrait supprimer la virgule qui marque l'apposition - mais dépend de la propriété de la valise de démonstration surchargée qui a la taille d'une «presque-malle ». Cette indication n'aurait pas de pertinence dans le genre de la recette. Cela signifie que bien qu'il s'apparente à ce genre de discours, ce texte veut par conséquent dire davantage. En effet, par ce mode d'écriture, l'auteur-narrateur fait naitre de la compassion non seulement à l'égard de Joseph dont on comprend qu'il ait pu mourir prématurément à l'âge de 41 ans, mais également à son propre égard, lui, «l'orphelin dans sa douzième année ». Or on sait que cet événement cataclysmique fut décisif pour l'avenir de Jean et que son écriture devait servir, dans un premier temps, à ressusciter par les mots le corps du père trop tôt disparu ${ }^{28}$. On comprend alors que le détournement du genre de discours procédural (à travers le type particulier de la recette) et sa transmutation bakhtinienne dans le roman soient une manière d'idéaliser la personne du père, qui, conduite différemment, eût pu manquer son but. Je pense par exemple au genre rhétorique épidictique et au discours de laudation trop appuyé. L'auteur-narrateur fait ainsi preuve d'un art consommé de la persuasion, qui entrerait parfaitement dans le champ d'action de la nouvelle rhétorique, telle que la décrivent Perelman et Olbrechts-Tyteca ${ }^{29}$.

\footnotetext{
${ }^{26}$ Fløttum, K., 2002-a: 9.

${ }^{27} \mathrm{Je}$ souligne par l'italique.

${ }^{28}$ Cf. Freyermuth, S., 2006 et Freyermuth, S., 2011.

${ }^{29}$ Perelman, C. et Olbrechts-Tyteca, L., [1988, 1992], 2000.
} 
Un genre peut en cacher un autre : une histoire de détournement

\section{Conclusion}

Pour en revenir à la question initiale de l'interaction qui devrait nouer les genres de discours aux faits de langue, il semble que dans le cas exposé ci-dessus, l'approche $\mathrm{du}$ texte romanesque à travers ceux-ci soit un préalable indispensable. Mais on admettra aussi qu'on n'interroge le texte d'une manière plus pointue qu'en fonction de la présence d'indices inhabituels dans le contexte dans lequel ils se présentent; pour reprendre les termes de Riffaterre ${ }^{30}$, on a affaire en l'occurrence à un effet de contraste (dû aux éléments qui débordent du cadre du texte procédural) et à un autre de convergence (les faisceaux de critères atypiques). En outre, lorsque des propriétés nous paraissent pertinentes et suffisantes pour permettre l'identification d'un genre, il est prudent de s'interroger malgré tout sur les effets pragmatiques de l'énoncé. On pourra alors se rendre compte qu'il existe des catégories masquées qui empruntent par détournement telle ou telle apparence, mais qui agissent en réalité comme un autre genre de discours, défini par des critères bien différents.

30 Riffaterre, M., 1959. 
Sylvie Freyermuth

\section{BIBLIOGRAPHIE}

ADAM, J.-M., [1992] - 20012, Les textes : types et prototypes, Paris, Nathan-Université.

ADAM, J.-M., 1997, Le style dans la langue. Une reconception de la stylistique, Lausanne-Paris, Delachaux et Niestlé, chapitre «Sciences des discours », voir p. 55 et sq.

ADAM, J.-M., 2002, Langue française, $\mathrm{n}^{\circ}$ 135, « Le style dans la langue et dans les textes », p. 7194.

Bakhtine, M., (Volochinov), 1977, Le marxisme et la philosophie du langage. Essai d'application de la méthode sociologique en linguistique, Paris, Les éditions de minuit.

BAKHTine, M., 1984, Esthétique de la création verbale, Paris, Gallimard.

BALLY, Ch., [1909], 1951, Traité de stylistique française (2 vol.), Genève-Paris, Georg et Klincksieck.

BALLY, Ch., [1913], 1965, Le langage et la vie, Genève, Droz.

BeEtham, G., FernándeZ, M., 2010, «Inter/Trans/Post-Disciplinarity : Explorations of Encounters Across Disciplines », Graduate Journal of Social Science, 7, 1, p. 7-13.

DESPRÉs, C., LAWRENCE, R. J., 2004, «Introduction », Futures, 36, p. 397-405.

Ducrot, O., 1984, Le dire et le dit. Paris, Les Éditions de Minuit.

FløtтuM, K., 1999, «Typologie textuelle et polyphonie: quelques questions», Tribune, 9, Universitetet i Bergen, http://www.hum.au.dk/romansk/polyfoni/Tribune9

FLøTTUM, K., 2002, «La polyphonie dans une perspective macro-sémantique », Macro-syntaxe et macro-sémantique, Actes du colloque international d'Arbus, 17-19 mai 2001, Berne, Peter Lang, p. 337-359.

Fløtтum, K., 2002, «Polyphonie au niveau textuel», Romansk Forum, 16 - 2002 / 2, XV Skandinaviske romanistkongress, Oslo, 12-17 august 2002, p. 339-350.

Fløtтum, K., 2002, «Polyphonie et typologie revisitées », Polyfoni Arbejdspapirer, Séminaire de Bergen, 5, 19-20 avril 2002, p. 1-30.

Freyermuth, S., 2006, Jean Rouand et le périple initiatique: une poétique de la fluidité, Paris, L’Harmattan, Coll. « Critiques littéraires ».

FrEYERMUTH, S., 2007, «Encodage et décodage du pronom ana-cataphorique: réflexion stylistique sur un outil de cohésion romanesque dans l'œuvre de Jean Rouaud », Actes $d u$ Colloque international Littérature et linguistique : diachronie / synchronie — autour des travaux de Michèle Perret', CD- ROM, D. Lagorgette et M. Lignereux (dir.), Chambéry, Université de Savoie, p. 352-363.

Freyermuth, S., 2010, «Du 'Cycle de Minuit' à la liberté du romancier : la permanence et le mouvement dans l'écriture roualdienne », P. Marillaud et R. Gauthier (éds.), Ecritures évolutives: entre transgression et innovation, C.A.L.S. / C.P.S.T., Toulouse, Université de Toulouse-Le Mirail, p. 37-46.

Freyermuth, S., 2011, Jean Rouand et l'écriture «les yeux clos». De la mémoire engagée à la mémoire incarnée, Paris, L'Harmattan, Coll. « Critiques littéraires ». 
Grice, H. P., 1975, "Logic and Conversation », P. Cole \& J. L. Morgan (Eds.), Syntax and Semantics, vol. 3, Speech Acts, New York, Academic Press, p. 41-58 ; traduction, 1979, «Logique et conversation », Communications, 30, p. 57-72.

Heurley, L., 2001, «Compréhension et utilisation de textes procéduraux : l'effet de l'ordre de mention des informations », Revue Française de Linguistique Appliquée, 2001/2, Volume VI, p. 29-46.

HuUtoniem, K., ThOmpson-KLeIN, J., BruUn, H., HukKINEN, J., 2010, « Analyzing Interdisciplinarity: Typology and Indicators », Research Policy, 39, p. 79-88.

JACQUES, M.-P., POIBEAU, Th., «Étudier des structures de discours : préoccupations pratiques et méthodologiques », Corela, cognition, représentation, langage, 8, Publié en ligne le 29 novembre 2010, n.p.

KLEIBER, G., 1994, "Contexte, interprétation et mémoire : Approche standard vs. approche cognitive », Langue française, 103 , p. 9-22.

KLEIBER, G., 1994, Anaphores et pronoms, Louvain-La-Neuve, Duculot, Coll. « Champs linguistiques ».

KLEIBER, G., 1997, «Référents évolutifs et pronoms : une suite », dans G. Kleiber, C. Schnedecker et J.-E. Tyvaert, (éds.), La continuité référentielle, Recherches linguistiques, 20, Metz, p. 115-148.

KLEIBER, G., 2002, «Micro-anaphorique et macro-anaphorique avec une vue spéciale sur les noms de parenté en anaphore associative ", Macro-syntaxe et macro-sémantique, Actes $d u$ colloque international d'Arbus, 17-19 mai 2001, Berne, Peter Lang, p. 207-238.

Maingueneau, D., 2003, "Ouverture. Un tournant dans les études littéraires », Amossy, R. et Maingueneau, D., (éds.), L'analyse du discours, Toulouse, Presses Universitaires du Mirail, coll. « Théories de la littérature », « Cribles », p. 15-25.

Perelman, C. et Olbrechts-TyteCA, L., [1988, 1992], 2000, Traité de l'argumentation, Bruxelles, Éditions de l'Université.

Perrin, L. (éd.), 2006, Le sens et ses voix. Dialogisme et polyphonie en langue et en discours, coll. "Recherches linguistiques », 28, Metz, UPV-Metz.

RiffATERRE, M., 1959, « Criteria for style analysis », Word, 15, p. 154-174.

Rouaud, J., 1993, Des hommes illustres, Paris, Les Éditions de Minuit. 